\title{
Mechanism of spontaneous formation of stable magnetic structures on the Sun
}

\author{
I. N. Kitiashvili ${ }^{1,2}$, A. G. Kosovichev ${ }^{1}$, A. A. Wray ${ }^{3}$, N. N. Mansour ${ }^{3}$ \\ ${ }^{1}$ Hansen Experimental Physics Laboratory, Stanford University, Stanford, CA 94305, USA \\ ${ }^{2}$ NORDITA, Dept. of Astronomy, AlbaNova Univ. Center, SE 10691 Stockholm, Sweden \\ ${ }^{3}$ NASA Ames Research Center, Moffett Field, Mountain View, CA 94040, USA
}

\begin{abstract}
One of the puzzling features of solar magnetism is formation of long-living compact magnetic structures; such as sunspots and pores, in the highly turbulent upper layer of the solar convective zone. We use realistic radiative 3D MHD simulations to investigate the interaction between magnetic field and turbulent convection. In the simulations, a weak vertical uniform magnetic field is imposed in a region of fully developed granular convection; and the total magnetic flux through the top and bottom boundaries is kept constant. The simulation results reveal a process of spontaneous formation of stable magnetic structures, which may be a key to understanding of the magnetic self-organization on the Sun and formation of pores and sunspots. This process consists of two basic steps: 1) formation of small-scale filamentary magnetic structures associated with concentrations of vorticity and whirlpool-type motions, and 2) merging of these structures due to the vortex attraction, caused by converging downdrafts around magnetic concentration below the surface. In the resulting large-scale structure maintained by the converging plasma motions, the magnetic field strength reaches $\sim 1.5 \mathrm{kG}$ at the surface and $\sim 6 \mathrm{kG}$ in the interior; and the surface structure resembles solar pores. The magnetic structure remains stable for the whole simulation run of several hours with no sign of decay.
\end{abstract}

Subject headings: sunspots — Sun: magnetic fields

\section{Introduction}

Sunspots and pores represent one of the oldest and most intriguing problem of solar magnetism. Despite the long history of observational and theoretical investigations (e.g.

\footnotetext{
${ }^{1}$ e-mail: irinasun@stanford.edu
} 
Bray \& Loughhead 1964; Moore \& Rabin 1985; Brandenburg et al. 2010) the mechanism of their formation is still open. Our understanding of MHD processes on the Sun is getting significantly improved with the rapid progress in observational instruments, data analysis, methods and numerical modeling. For example, the data obtained by helioseismology have provided initial information about the structure and dynamics of convective flows around sunspots and emerging magnetic flux beneath the solar surface (e.g. Kosovichev et al. 2000; Zhao et al. 2001; Kosovichev 2009). The high-resolution observations from ground-based telescopes and Hinode space mission have provided detailed data about the filamentary magnetic structures and flow dynamics on the surface (e.g. Ichimoto et al. 2007; Bonet et al. 2008; Attie et al. 2009; Balmaceda et al. 2010). In addition, important support for the understanding and interpretation of the observations is given by "realistic" radiative MHD numerical simulations, which are based on the first principles and take into account all essential physical processes. The recent progress in the numerical modeling has made it possible to reproduce in simulations many observational effects in the quiet Sun region, sunspots and active regions (e.g. Stein \& Nordlund 2001; Schüssler \& Vögler 2006; Jacoutot et al. 2008a,b; Martínez-Svkora et al. 2008; Kitiashvili et al.|2009a, 2010), magnetic flux emerging (e.g. Shibata et al. 1989; Cheung et al. 2008; Stein et al. 2009) and even the whole magnetic structures, such as pores and sunspots (e.g. Knölker \& Schüssler 1988; Stein et al. 2003; Bercik et al. 2003; Rempel et al. 2009). However, most of the modeling has been done by setting up the initial conditions with already existing magnetic structures, e.g. a horizontal flux tube for the modeling of magnetic flux emerging, or a vertical flux tube with strong field for the sunspot/pore structures simulations. It seems that so far only one study succeeded in reproducing a spontaneous formation of a micropore-like magnetic structure from an initially uniform field in the turbulent convection of the Sun (Stein et al. 2003). However, the lifetime of this structure was rather short, only "few convective turnover time scales" (Bercik et al. 2003). Similar calculations by Vögler et al. (2005) for a substantially shallower convective layer did not show the structure formation.

Here, we present new results of the realistic MHD simulations that show a process of spontaneous formation of a stable pore-like magnetic structure from an uniform magnetic field, and discuss the physical mechanism of the structure formation, and its dynamics and evolution.

\section{Numerical setup}

For the simulations we used a 3D radiative MHD code, "SolarBox", developed by A. Wray at NASA Advanced Supercomputing Division (Jacoutot et al. 2008a, b. bitiashvili et al. 
$2009 \mathrm{~b})$. The code is built for 3D simulations of compressible fluid flows in a magnetized and highly stratified medium of top layers of the convective zone and the low atmosphere, in the rectangular geometry. The code has been thoroughly tested and compared for test runs with a similar code of Stein \& Nordlund (2001). The code is based on the Large-Eddy Simulation (LES) approach, and solves the grid-cell averaged equations of the conservation of mass, momentum and energy. It takes into account the real-gas equation of state, ionization and excitation of all abundant species, and magnetic effects. A unique feature of the code is implementation of various sub-grid scale turbulence models. For this particular simulations we use the minimal hyperviscosity model (Jacoutot et al. 2008a).

The simulation results are obtained for the computational domain of $6.4 \times 6.4 \times 5.5 \mathrm{Mm}$ with the grid sizes: $50 \times 50 \times 43 \mathrm{~km}, 25 \times 25 \times 21.7 \mathrm{~km}$ and $12.5 \times 12.5 \times 11 \mathrm{~km}\left(128^{2} \times 127\right.$, $256^{2} \times 253$ and $512^{2} \times 505$ mesh points). The domain includes a top, $5 \mathrm{Mm}$-deep, layer of the convective zone and the low atmosphere. The lateral boundary conditions are periodic; and the top and bottom boundaries were closed for flows and maintain the constant total magnetic flux. The results have been verified by increasing the computational domain size to 12.8 $\mathrm{Mm}$ in the horizontal directions. The initial uniform magnetic field of various strength 1, 10 and $100 \mathrm{G}$, was superimposed over the fully developed granular convection. The computation runs were up to 8 hours of solar time.

We describe first the modeling of the solar convection without magnetic field, which prepares the initial conditions, and then, the simulations with the superimposed weak vertical magnetic field.

\subsection{Simulation of the quiet Sun regions}

Figure 1 shows snapshots for temperature (left column) and density (right) at the surface for the case without magnetic field. As usual, the convective motions develop a characteristic granulation pattern with the relatively hot and less dense upflowing plasma in the middle of the granular cells, and the lower temperature and higher density downflowing plasma at the intergranulation boundaries (dark lines of granulation). An interesting feature of the convective flows is the formation of whirlpool-like motions of different sizes $(\sim 0.2-1 \mathrm{Mm})$ and lifetimes $(\sim 15-20 \mathrm{~min} 1)$ at the vertexes of the intergranular lanes. The vortical motions are particularly well seen in the density variations. The centers of the whirlpools are seen as dark dots (indicated by white arrows in Fig. 1b) in the intergranular space. The evolution of

\footnotetext{
${ }^{1}$ The lifetime can be longer. It is difficult to estimate it accurately because during its evolution a vortex can significantly change the shape and size, and sometimes almost disappear and then rise again.
} 
these vortices is ultimately related to the dynamics of convective motions in the domain. The convective flows may sometime collect swirls in a local area, then merge and destroy them. Such vortical structures in simulations were first described by Stein \& Nordlund (1998). They showed that stronger vortices usually correlate with downflows, and this is also found in our results.

From time to time, convection creates pretty big whirlpools, as the one indicated by square in Fig. 1, which can swallow up other smaller swirls around them. The big swirls are usually easy to see also in the surface temperature and intensity variations. The detailed structure of a large whirlpool is shown in Figs 1 1 -f. The whirlpool structure is characterized by: 1) formation of "arms" of higher density that correlates with lower temperature; 2) a pronounced vortical structure of the velocity flow; 3) increased magnitude of the horizontal velocity up to $7-9 \mathrm{~km} / \mathrm{s}$; 4) a sharply decreased density in the central core of the vortex, and a slightly higher temperature than in the surrounding. The typical depth of large swirls is about $100-200 \mathrm{~km}$. Inside the whirlpool shown in Fig. 11, we can see a higher temperature tube-like structure, but it is unstable (in comparison with the whirlpool lifetime), and can be destroyed during the swirling motions.

The vortical motions in the solar granulation have been detected in high-resolution observations (e.g. Pötzi \& Brandt 2005), and the observational results generally agree with the simulations. In particular, recent observations of a quiet region, near the solar disk center detected magnetic bright points following a logarithmic spiral trajectory around intergranular points and engulfed by a downdraft (Bonet et al. 2008). The observations were interpreted as vortical flows that affects the bright point motions. These whirlpools have the size $\lesssim 0.5 \mathrm{Mm}$ and the lifetime of about $5 \mathrm{~min}$, without preferred direction of rotation. The distribution of vortices studied from the ground (Wang et al. 1995; Pötzi \& Brandt 2005, 2007; Bonet et al. 2008) and space observations (Attie et al. 2009) shows strong preferences to concentration in regions of downflows, particulary at the mesogranular scale (Pötzi \& Brandt 2005, 2007). Our simulations for the domain of the horizontal size of $12.8 \mathrm{Mm}$ also show a tendency of concentration of vortices on a mesogranular scale. We plan to discuss this effect in a separate paper. Here we focus on the links between the whirlpools and magnetic structure formation.

\subsection{Spontaneous formation of magnetic structures}

To investigate the process of magnetic field structuring in the turbulent convective plasma we made a series of simulations for the initial vertical uniform magnetic field, $B z_{0}$, varying from 1 to $100 \mathrm{G}$, different computational grids and domain sizes. Qualitatively 
the simulation results are very similar in all these cases, and show the formation of stable magnetic pore-like structures. In Figures 2 and 3 we present the results for the case of $B z_{0}=100 \mathrm{G}$, the grid size of $25 \mathrm{~km}$, and the domain size of $6.4 \times 6.4 \times 5.5 \mathrm{Mm}$, for which we have made the longest run ( $\gtrsim$ hours). The periodic lateral boundary conditions allow us, for the illustration purpose, to shift the horizontal frame so that the structure is located close to the center. As we see in the simulations, the structure can be formed in any place of our computational domain, but usually the process starts at one of the strongest vortices.

Figure 2 shows snapshots of the vertical magnetic field (background color image), horizontal flow field (arrows), and the vorticity magnitude (black contour lines) for four moments of time: 3, 10, 20 and $60 \mathrm{~min}$ after the moment $t=0$, when the $100 \mathrm{G}$ vertical field was uniformly distributed in the computational domain. During few minutes the magnetic field is swept into the intergranular lines, and is significantly amplified up to $\sim 500-1000 \mathrm{G}$. The vortices and magnetic field get concentrated at some locations in the intergranular lanes, where they are deformed and became elongated (or elliptically shaped) along the intergranular lines. The process of formation of a large-scale magnetic structure starts at a strongest vortex in our domain. Since our computational domain is periodic we choose the horizontal coordinates in such a way that this vortex is located in the middle of Figure 2a. This large swirl sweeps magnetic field and also becomes stretched by strong horizontal shear flows. The whirlwind causes deformation of the intergranular space, and creates a cavity of low density, temperature and pressure. The cavity expands and increases the accumulation of magnetic field (Fig. 2a). A similar process of magnetic field concentration, sweeping, twisting and stretching by vortical motions in the intergranular lane was initially observed in the simulations of Stein et al. (2003).

During the next few minutes the deformation of the "parent" vortex continues; then it gets destroyed on the surface by $t=10 \mathrm{~min}$ (Fig. 2 b), but leaves strong downdraft motions in the interior (Fig. $3 \mathrm{~b}$ ). The process of accumulation of magnetic flux in this area continues. The local concentrations of magnetic field and vorticity get stronger and are moved by convective motions in the direction of the initial cavity, into the region where the gas pressure remains systematically low due to the downdrafts. In Fig. 2b several smallscale structures can be seen in this area. However, it is difficult to recognize the center of the attraction on the surface. Collisions of flows coming from different directions create additional vortices, and this seems to accelerate the accumulation process. As a result, the different small magnetic structures join together in a magnetic conglomerate that continues to attract other magnetic micro structures (Fig. 2kc) and becomes more compact (Fig. 22d). 
This process of the magnetic structure formation is particularly well illustrated in the movie2. It shows a correlation between the distribution of vortices and the areas with concentrated magnetic field elements, as well as the mutual influence of convection, vortices and magnetic field.

Figure 3 shows a vertical slice for the same moments of time. The arrows show the velocity field calculated from the $x$ - and $z$-components. Thus, we can see that the initially uniform magnetic field has local concentrations in the regions of the near-surface downdraft, and has a very fragmentary structure during the first few minutes (Fig. 3a). The continuing and extending into the deeper layers downflows are accompanied by the local concentrations of the magnetic field strength (Fig. 3b), which grows after $\sim 20$ minutes to $\sim 2 \mathrm{kG}$ below the surface (Fig. 3r). By $t=60 \mathrm{~min}$, the magnetic field is mostly concentrated in a single flux structure with a maximum field strength of about $\sim 4 \mathrm{kG}$ at the depth of $\sim 1-$ $4 \mathrm{Mm}$, and $\sim 1.4-1.5 \mathrm{kG}$ at the surface (Fig. $3 \mathrm{~d}$ ). The magnetic field is weaker and more disperse near the bottom of our domain, which is impenetrable for flows. The flux-tube interior represents a cluster-type structure (Figs. 2d, 3 $\mathrm{d}$ and 4), as initially predicted by Parker (1979) and observed on a larger scale for a sunspot by helioseismology (Zhao et al. 2010). In our simulations the cluster structure is represented by internal field concentrations (flux tubes), $100-200 \mathrm{~km}$ thick, in which the field strength reaches $6 \mathrm{kG}$ after 1 hour (Fig. 4a). The velocity distribution shows strong, often supersonic, downflows around the magnetic structure. Inside the magnetic structure the convective flows are suppressed by strong magnetic field. However, despite the weak velocities $(\sim 0.1-0.2 \mathrm{~km} / \mathrm{s})$ there are very small elongated convective cells resembling the umbral dots observed at the surface. The distribution of density fluctuations (Fig. 4b) shows the following basic properties: a) decrease of density inside the magnetic structure; b) fine needle-like structurization; c) a thin near-surface layer of slightly higher density; and d) higher density around the structure, particularly in the deep layers of the domain (Fig. 4b). We have followed the evolution of the magnetic pore-like structure for more than 8 solar hours, and did not see any indication of its decay. However, the shape and other properties fluctuate during this evolution. The decay of this structure is probably prevented by keeping the total magnetic flux constant during the run. We repeated the simulation when the initial uniform magnetic field was introduced at the different moment of time. In one case we observed the formation of two separate magnetic structures, which later merged together, but the whole process was very similar.

\footnotetext{
${ }^{2}$ See also movie: http://soi.stanford.edu/ irina/fig2swirls_movie.mpeg
} 


\section{Conclusions}

Initially, the vortical motions in quiet-Sun regions were detected on a large scale of $\sim 5 \mathrm{Mm}$ (Brandt et al. 1988). With the development of instrumentation it became possible to observe small-scale swirls in the photosphere (Bonet et al. 2008) (with size $<0.5 \mathrm{Mm}$ ) and also in the chromosphere 1.5 Mm (Wedemeyer-Böhm \& Rouppe van der Voort 2009). Very recently, the high-resolution observations revealed a process of dragging of small-scale magnetic concentrations toward the center of a convective vortex motion in the photosphere (Balmaceda et al. 2010).

Our simulations show that the small-scale vortices representing whirlpool-type motions at intersections of the intergranular lanes may play important roles in the dynamics of the quiet-Sun and magnetic regions. Our simulations show that the process of formation of smallscale magnetic structures and their accumulation into a large-scale magnetic structure is associated with strong vortical downdrafts developed around these structures. The resulting stable pore-like magnetic structure has the highest field strength of $\sim 6 \mathrm{kG}$ at the depth of $1-4 \mathrm{Mm}$ and $\sim 1.5 \mathrm{kG}$ at the surface. It has a cluster-like internal structurization, and seems to be maintained by strong downdrafts converging around this structure and extending into the deep layers. Our simulations show that this internal dynamics plays a critical role in the magnetic self-organization of solar magnetic fields and formation of large-scale magnetic structures.

\section{REFERENCES}

Attie, R., Innes, D. E. \& Potts, H. E. 2009, A\&A, 493, L13.

Balmaceda, L., Vargas Domnguez, S., Palacios, J., Cabello, I. \& Domingo, V. 2010, A\&A, in press. arXiv:1004.1185

Bercik, D. J., Nordlund, Å. \& Stein,R. F. 2003, Proc. of SOHO 12/GONG+ 2002 "Local and Global Helioseismology: the Present and Future", 201.

Bonet, J. A., Márquez, I., Sánchez Almeida, J., Cabello, I. \& Domingo, V. 2008, ApJ, 687, L131.

Brandenburg, A., Kleeorin, N. \& Rogachevskii, I. 2010, Astronomische Nachrichten, 331, 5.

Brandt, P. N., Scharmer, G. B., Ferguson, S., Shine, R. A., Tarbell, T. D. \& Title, A. M. 1988, Nature, 335, 238. 
Bray, R. J. \& Loughhead, R. E. 1964, Sunspots. Dover publications, New York.

Cheung, M.C.M., Schüssler, M., Tarbell, T. D. \& Title, A. M. 2008, ApJ, 687, 1373.

Cameron, R. Schüssler, M., Vögler, A. \& Zakharov, V. 2007, A\&A, 474, 261.

Ichimoto, K., Shine, R. A., Lites, B., Kubo, M., Shimizu, T., et al. 2007, PASJ, 59, S593.

Jacoutot, L., Kosovichev, A. G., Wray, A. A. \& Mansour, N. N. 2008a, ApJ, 682, 1386.

Jacoutot, L., Kosovichev, A. G., Wray, A. A. \& Mansour, N. N. 2008b, ApJ, 684, L51.

Kitiashvili, I. N., Kosovichev, A. G., Wray, A. A. \& Mansour, N. N. 2009a, ApJ, 700, L178.

Kitiashvili, I. N., Kosovichev, A. G., Wray, A. A. \& Mansour, N. N. 2009b, Proc. of ParCFD Conf., NASA Ames, 424.

Kitiashvili, I. N., Bellot Rubio, L. R, Kosovichev, A. G., Mansour, N. N., Sainz Dalda, A. \& Wray, A. A. 2010, ApJ, submitted.

Knölker, M. \& Schüssler, M. 1988, A\&A, 202, 275.

Kosovichev, A. G., Duvall, T. L., Jr. \& Scherrer, P. H. 2000, Solar Physics, 192, 159.

Kosovichev, A. G. 2009, Space Sci.Rev., 144,175.

Martínez-Sykora, J., Hansteen, V. \& Carlson, M. 2008, ApJ, 679, 871.

Moore, R. \& Rabin, D. 2003, Annual review of astronomy and astrophysics, 23, 239.

Parker, E. N. 1979, ApJ, 230, 905.

Pötzi, W. \& Brandt, P. N. 2005, Hvar Obs. Bull., 29, 61.

Pötzi, W. \& Brandt, P. N. 2007, Cent. Eur. Astrophys. Bull., 31, 11.

Rempel, M., Schüssler, M., \& Knölker, M. 2009, ApJ, 691, 640.

Schüssler, M. \& Vögler, A. 2006, ApJ, 641, L73.

Shibata, K., Tajima, T., Steinolfson, R. S. \& Matsumoto, R. 1989, ApJ, 345, 584.

Stein, R. F., Bercik, D. \& Nordlund, A. 2003, ASP Conf.Series, 286, 121.

Stein, R. F., Lagerfjärd, A., Nordlund, A. \& Georgobiani, D. 2009, arXiv:0912.4938.

Stein, R. F. \& Nordlund, A. 1998, ApJ, 449, 914. 
Stein, R. F. \& Nordlund, A. 2001, ApJ, 546, 585.

Vögler, A., Shelyag, S., Schüssler, M., Cattaneo, F., Emonet, T. \& Linde, T. 2005, A\&A, $429,335$.

Wang, Y., Noyes, R. W., Tarbell T. D. \& Title, A. M. 1995, ApJ, 447, 419.

Wedemeyer-Böhm, S. \& Rouppe van der Voort, L. 2009, A\&A, 507, L9.

Zhao, J., Kosovichev, A. G. \& Duvall, T. L. 2001, ApJ, 557, 384.

Zhao, J., Kosovichev, A. G. \& Sekii, T. 2010, ApJ, 708, 304.

This preprint was prepared with the AAS IATEX macros v5.2. 

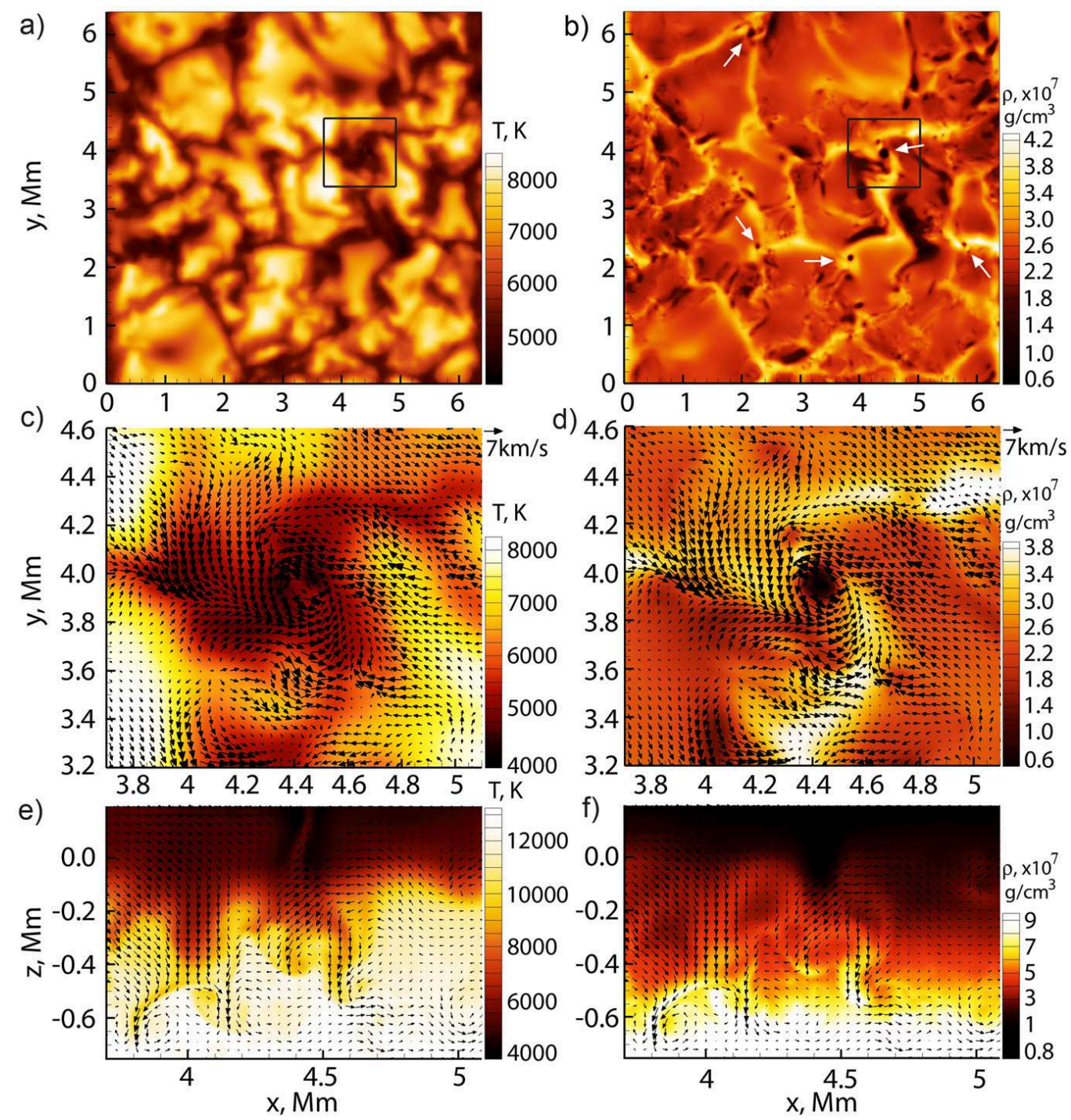

Fig. 1. - Snapshots of granular convection at the surface for the simulations without magnetic field, and the horizontal resolution of $12.5 \mathrm{~km}$ : temperature (left column) and density (right). The black square indicates a large whirlpool, the horizontal and vertical structure of which are shown in panels c) - f). Black arrows show the flow velocity. White arrows in panel b) point to the centers of some vortices (dark low-density points). 

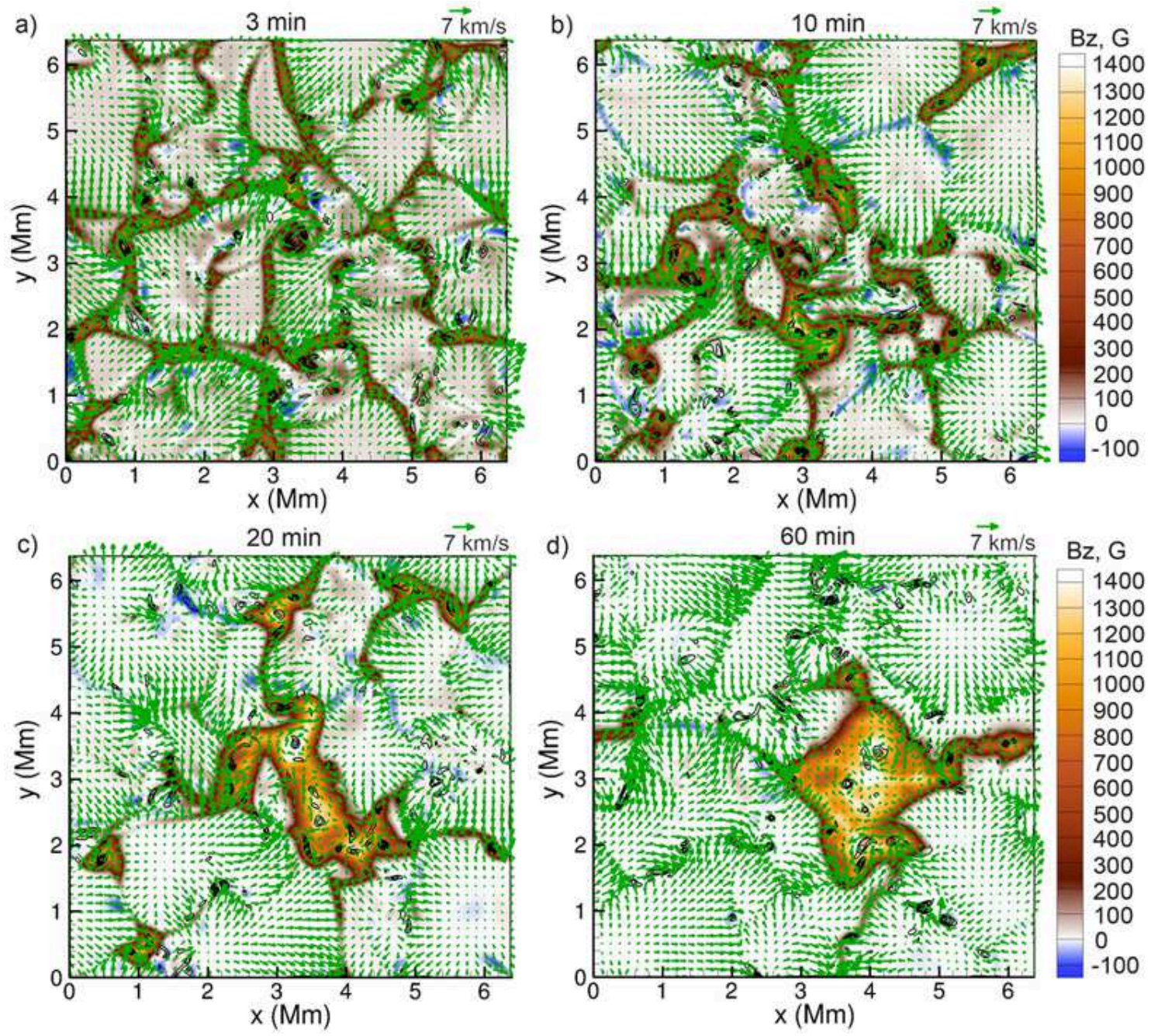

Fig. 2.- Snapshots of the surface distribution of vertical magnetic field (color background), horizontal flows (arrows) and vorticity magnitude (black contour lines) for four moments of time: 3, 10, 20 and $60 \mathrm{~min}$, from the moment of initiation of a uniform magnetic field $\left(B z_{0}=100 \mathrm{G}\right)$. 

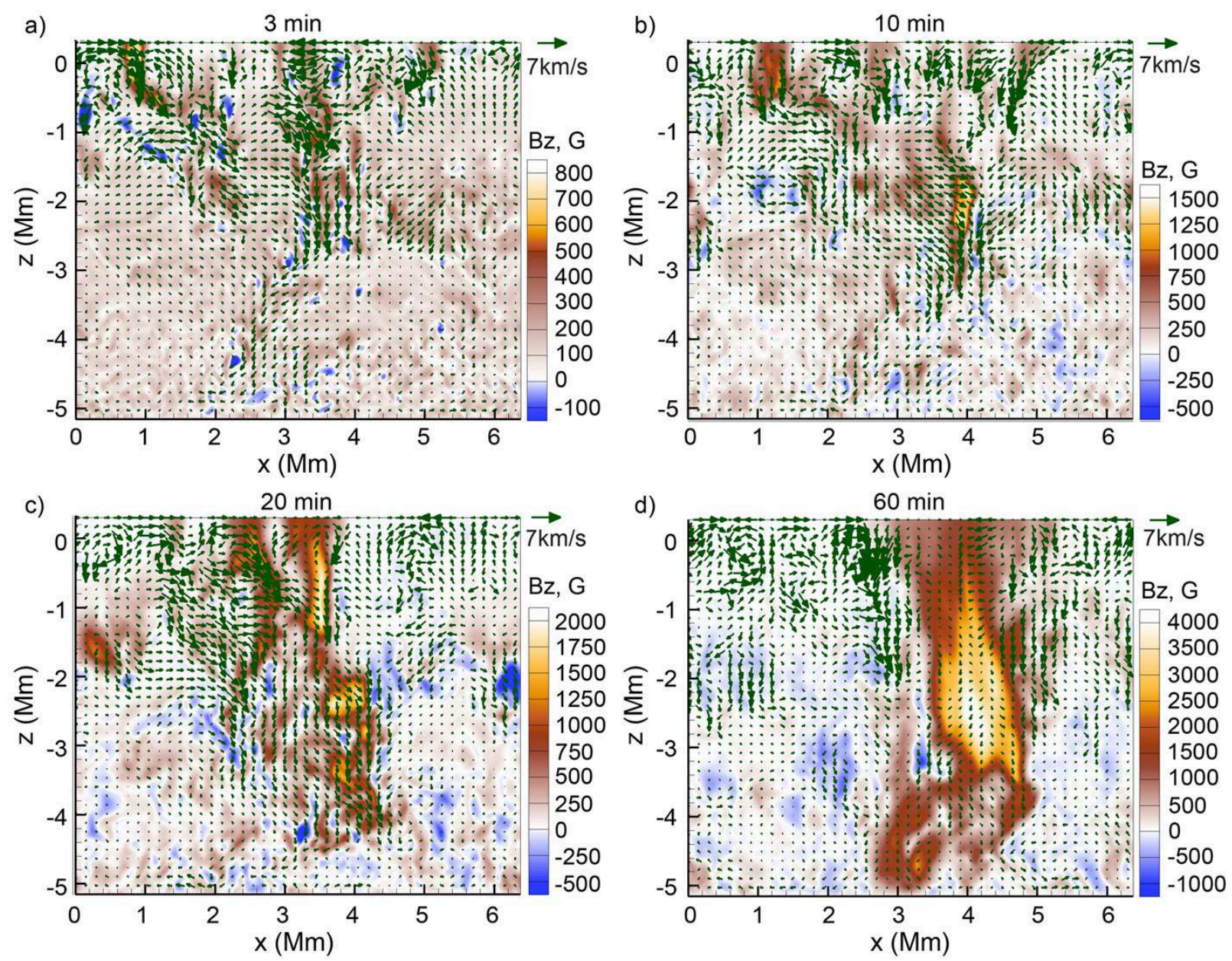

Fig. 3.- Vertical snapshots of the vertical magnetic field (color background) and the horizontal flows (arrows) for $t=3,10,20$ and $60 \mathrm{~min}$. 

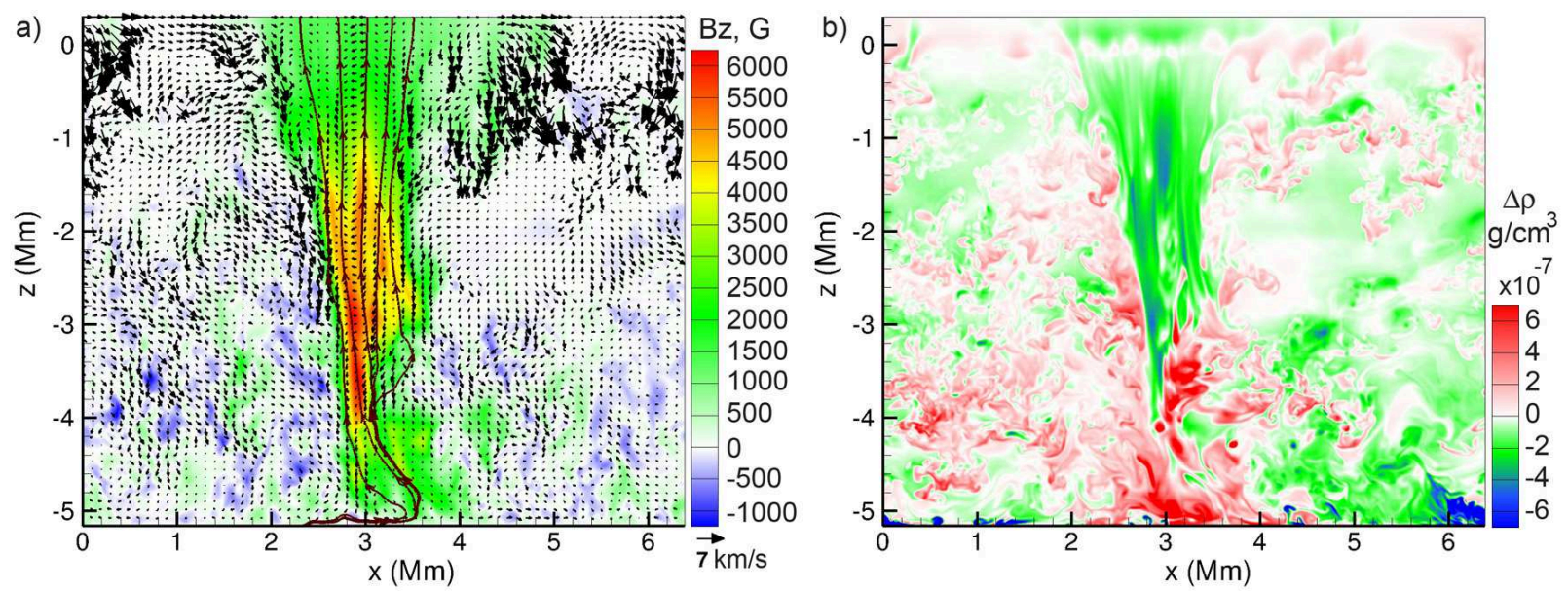

Fig. 4. - Vertical slices though the magnetic structure at $\mathrm{t}=84 \mathrm{~min}$ for the $12.5 \mathrm{~km}$ resolution: a) the vertical component of magnetic field (color background), flow velocity (arrows), magnetic field lines (contour lines); and b) variations of density with respect to a mean density profile of the convection simulations without magnetic field. The velocity vectors and magnetic field lines in panel a) are calculated from the corresponding $x$ - and $z$-components. 